\section{Relación entre ruido, estrés térmico con el comportamiento auditivo en trabajadores de Mina}

Relationship between noise, thermal stress and auditory behavior in Mina workers

César Fredy Toledo Cubillos

Corporación Universitaria Minuto de

Dios, Facultad Ciencias Empresariales,

Programa Académico: Administración

en Seguridad y Salud en el

Trabajo Nombre Semillero de

Investigación: SIVIEPI

https://orcid.org/0000-0003-3945-

1557

cesar.toledo.c@uniminuto.edu,

Bogota - Colombia

Luis Miguel Cárdenas Castellanos

Corporación Universitaria Minuto de

Dios, Facultad Ciencias

Empresariales, Programa Académico:

Administración en Seguridad

y Salud en el Trabajo Nombre

Semillero de Investigación: SISOLUX.

https://orcid.org/0000-0002-1526-

6763

Luis.cardenasc@uniminuto.edu

http://www.jah-

journal.com/index.php/jah

Journal of American health, July -

December vol. 1. Num. $2-2018$

Esta obra está bajo una Licencia

Creative Commons

Atribución-NoComercial-

CompartirIgual 4.0 Internacional.

RECIBIDO: 15 DE MARZO 2018

ACEPTADO: 11 DE MAYO 2018

PUBLICADO: 3 DE JULIO 2018

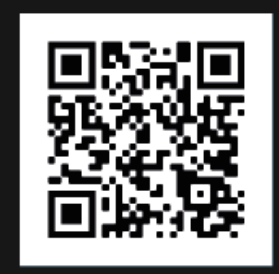

scan this QR

code with your

smart phone or

mobile device to

read more papers

\section{RESUMEN}

Es una investigación cuantitativa correlacional de corte transversal que buscó determinar la relación entre los factores de riesgos físicos como la temperatura, el ruido, y el comportamiento del umbral auditivo en 24 trabajadores de una mina a cielo abierto ubicada en Nilo Cundinamarca, unos con exposición directa a ruido laboral y temperatura, otros sin exposición. Los datos se recolectaron mediante encuestas del perfil sociodemográfico, aplicación de medición de sonometrías, el índice de estrés térmico (WBGT) y las audiometrías; estás mediciones se realizaron durante tres momentos de la jornada laboral: 7: 00 a.m, 12:00 p.m. y 5:00 p.m. En los resultados se observa una correlación significativa entre el tiempo de exposición a ruido ( 6 horas) con una media para los expuestos de $(87,5$ $\mathrm{dB})$, el índice de estrés térmico $\left(31,4^{\circ} \mathrm{C}\right), \mathrm{y}$ el cambio del umbral auditivo en las frecuencias agudas entre 5 y $10(\mathrm{~dB})$, en la última medición después de 10 horas exposición se percibe un cambio en el umbral auditivo de las frecuencias 4000 a $8000 \mathrm{~Hz}$ entre 10 y 15 (dB), mientras el grupo control no expuestos no se encontró cambios significativos en el umbral auditivo durante las 10 horas de seguimiento.

PALABRAS CLAVE: Umbral Auditivo, Índice de estrés térmico, sonometría, Ruido, Temperatura

\section{ABSTRACT}

It is a cross-sectional quantitative crosssectional investigation that sought to determine the relationship between physical risk factors such as temperature, noise, and auditory threshold behavior in 24 workers of an open pit mine located in Nilo Cundinamarca, some with direct 
exposure at work noise and temperature, others without exposure. Data were collected through sociodemographic profile surveys, sonometry measurement application, thermal stress index (WBGT) and audiometry; These measurements were made during three moments of the working day: 7:00 a.m., 12:00 p.m. and 5:00 p.m. The results show a significant correlation between the exposure time to noise (6 hours) with an average for those exposed $(87.5 \mathrm{~dB})$, the thermal stress index $\left(31.4^{\circ} \mathrm{C}\right)$, and the change in auditory threshold in the acute frequencies between 5 and $10(\mathrm{~dB})$, in the last measurement after 10 hours exposure a change in the auditory threshold of the frequencies 4000 to 8000 $\mathrm{Hz}$ between 10 and $15(\mathrm{~dB})$ is perceived, while the control group unexposed no significant changes were found in the auditory threshold during the 10 hours of follow-up.

KEY WORD: Auditory Threshold, Thermal stress index, sonometry, Noise, Temperature

\section{INTRODUCCIÓN}

El ruido laboral es algo consustancial con la mayoría de los trabajos operativo, dependiendo del tipo de herramientas, equipos, o maquinas que se manipulación producirán una serie de ruidos que afectan la salud auditiva de dichos trabajadores. Los roles ocupacionales de los operarios de las minas cielo abierto, conviven en un entorno agresivo para la conservación auditiva, dando lugar a una clara patología específica llamada Hipoacusia Neurosensorial. Los efectos nocivos del ruido sobre la audición se conocen desde hace siglos; Plinio el viejo, escribía como los esclavos de las minas que laboraban cerca de las cascadas del río Nilo iban perdiendo el sentido de la audición (Rosen, 1974).

En la llínea hermenéutica de la etiología de la sordera ocupacional que se mantiene hasta la actualidad como lo demuestra los estudios de la OMS, sse estima que 466 millones de personas en todo el mundo padecen pérdida de audición incapacitante, se calcula que, en 2050, más de 900 millones de personas-es decir, una de cada 10- sufrirá una pérdida de audición discapacitante; Los casos desatendidos de pérdida de audición representan un coste mundial anual de 750000 millones de dólares internacionales (Organización Mundial de la Salud, 2019). En un estudio realizado en Santiago de Chile en el 2013, en 37 trabajadores de la industria textil, con dos grupos uno de control no expuesto a ruido laboral y el otro con exposición, se determinó el umbral auditivo de los dos grupos por medio de la audiometría obteniendo resultados, que el $64,3 \%$ de los trabajadores expuestos a ruidos de alta intensidad presentaron audición normal, mientras que el 35,7\% presentaron trauma acústico, de los cuales el $80 \%$ se desempeñaba en el área de las máquinas de coser, cuyo ruido promedio fue de $95 \mathrm{~dB}$. (A) y el $20 \%$ restante, en corte con $90 \mathrm{~dB}$. (A) de ruido promedio, al contrastar el grupo control con el subconjunto del grupo estudio que presentó audición dentro de los rangos de normalidad (Silva, 2013).

En la tesis doctoral "Validez de los test predictivos de la fatiga auditiva en la prevención del trauma acústico" realizada en 1992 en Madrid, en la que se establece la relación si los test de fatiga auditiva (test de peyser, Tone Decay test) y los de reclutamiento auditivo (S.I.S.I. test, Lüscher) logran o no ser predictivos en la susceptibilidad de sufrir alguna pérdida auditiva, en la que afirma el investigador que la fatiga auditiva en la mayoría de veces causa un Cambio Transitorio del Umbral al finalizar la jornada laboral, y en menos proporción en otras personas 
dejan secuelas irreversibles en forma de Cambio Permanente del Umbral. En los resultados obtenidos en el estudio con militares, encontraron que el $79 \%$ del personal examinados con los test desarrollaron Cambio Transitorio del Umbral auditivo, y los demás Cambios permanentes del umbral (López, 1992).

Los trabajadores de la mina a cielo abierto ubicada en el departamento de Cundinamarca tienen como funciones extraer una serie de materias primas como granito, gravilla, arena, arcilla, entre otros; también conviven con una serie riesgos físicos de ruido y altas temperaturas generadas por el alto nivel de radiación solar de la región, en lo relacionado con ruido se producen por las fuentes como los automotores de las maquinarias pesadas, las plantas de trituración de material, la explotación del material de cantera, entre otros.

En las investigaciones realizadas con trabajadores de minería a cielo abierto en España, en la tesis doctoral Ignacio Pavón García (2007), estudió los niveles de ruido a los que se exponen los trabajadores del sector minero y las empresas de fabricación de cementos en la que utilizaron una serie de técnicas de medición de ruido, en los resultados obtenidos por el estudio con mediciones tradiciones con sonómetros y dosímetros arrojaron niveles altos de exposición sonora en el $90,2 \%$ de los puestos de trabajo con un LEx.8h superior de 80 (dBA), un $47,5 \%$ que supera los 90 (dBA) (García, 2007).

En la revisión de antecedentes en el estado del arte no se halló estudios correlacionales entre el índice de estrés térmico (WBGT) y la fatiga auditiva en la trabajadora fundamenten dicha relación causal, si se encontró una serie de investigaciones en la que se estudia los dos factores de riesgo de forma independiente sin correlacionar las variables; uno de ellos es el realizado en Ecuador, con una muestra 20 trabajadores aclimatados de una planta de generación eléctrica con una exposición 12 horas jornada laboral, en la que obtuvieron como resultado de dosimetría de $80,7 \mathrm{dBA}$, en el riesgo de estrés térmico en calderas de WBGT de $31^{\circ} \mathrm{C}$ con oscilación a $30,9^{\circ} \mathrm{C}$ (Soto, 2010 ).

En una investigación realizada en la Habana titulada "alteraciones auditivas en trabajadores expuestos al ruido industrial"; que buscó medir con audiometrías de control el grado de pérdida auditiva de un grupo de trabajadores con la finalidad de determinar el grado de exposición de los mismos, obtuvo como resultados que el 78,9\% de los trabajadores generaron hipoacusia por exposición al ruido laboral (Hernández \& González, 2008).

El presente estudio tuvo como propósito determinar si los agentes contaminantes de ruido y el nivel de estrés térmico influyen en el comportamiento auditivo en una jornada laboral de 10 horas en trabajadores de una mina a cielo abierto ubicada en el departamento de Cundinamarca.

\section{MATERIALES Y MÉTODOS}

Enfoque investigativo: cuantitativo.

Tipo de estudio: Se realizó un estudio correlacional-transversal.

Población de estudio: 36 trabajadores de una empresa minera a cielo abierto ubicada en el municipio de Nilo Cundinamarca.

Muestra: muestreo de conveniencia no probabilístico de 24 trabajadores de una la empresa minera a cielo abierto ubicada en el municipio de Nilo Cundinamarca; la mitad expuesta a ruido y carga térmica y la otra mitad no expuesta a dichos riesgos. 
Criterios de inclusión: Todo trabajador vinculado a la empresa minera que tenga una jornada laboral de 10 horas, con exposición a ruido laboral por encima de los $80 \mathrm{dBA}$, un índice de térmico WBGT por encima de los $30^{\circ} \mathrm{C}$; trabajadores administrativos no expuestos a ruido laboral con un nivel de exposición máximo de $65 \mathrm{~dB}$, y un índice de térmico WBGT de límite máximo de $29^{\circ} \mathrm{C}$.

Criterios de Exclusión: todos los trabajadores que laboran menos de las 10 horas de la jornada laboral.

Las etapas que comprendió el estudio:

Se aplicó a los trabajadores una serie de preguntas para obtener el perfil sociodemográfico, y las actividades extra-laborales con exposición a ruido; posteriormente se realizaron las mediciones de sonometría, índice de estrés térmico y audiometría según las normas técnicas en el siguiente orden cronológico como lo muestra la Figura 1. y Tabla 1.

Figura 1. Toma de sonometría y medición WBGT térmico
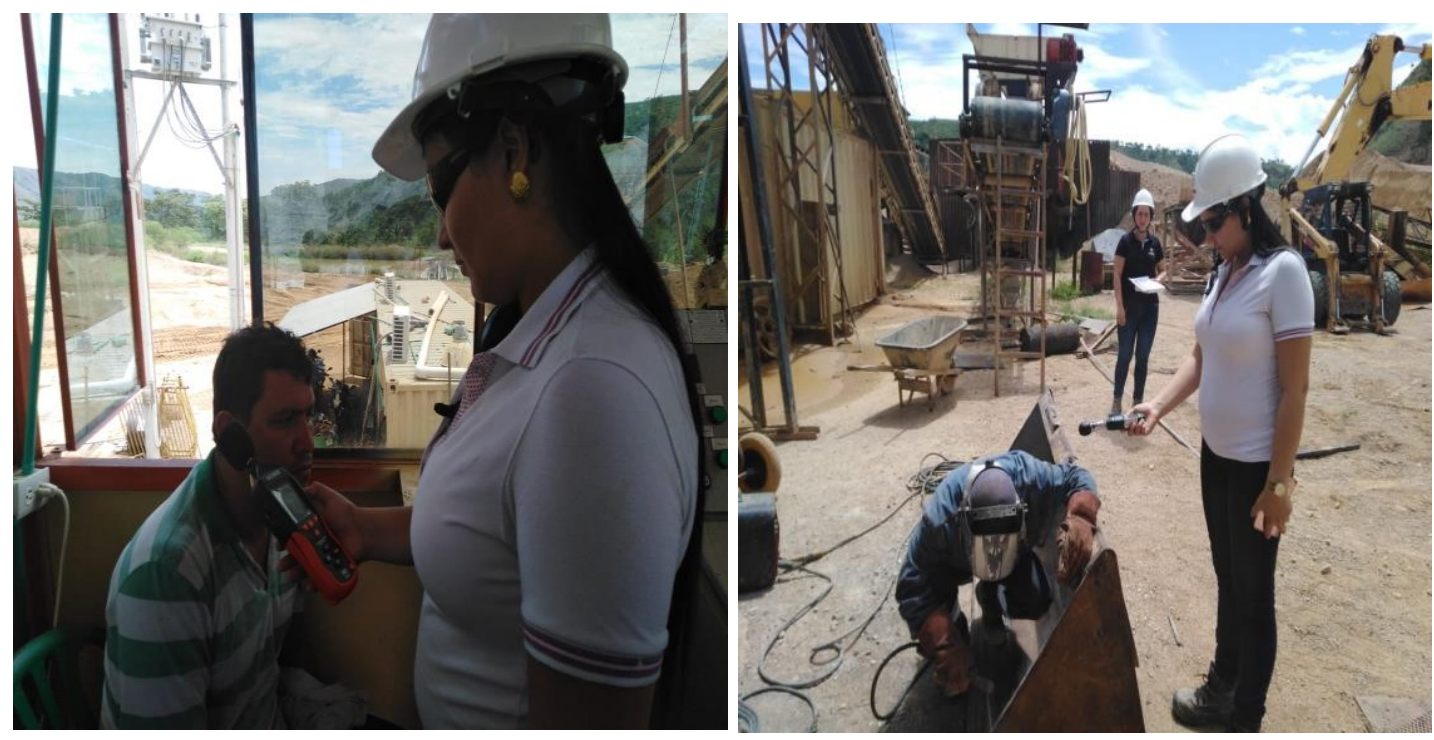

Fuente: Semillero SIVIEPI

Se realizó tres mediciones tamizaje de las audiometrías a los trabajadores en tres momentos de la jornada laboral, en la primera hora, en la sexta hora y la úúltima hora de trabajo (decima). Con base en los criterios se aplicaron los criterios técnicos SAL-AMA (Asociación Médica Americana) que emplea la OSHA, consiste en sacar el promedio de las frecuencias de lenguaje $(500,1000,2000$ y $3000 \mathrm{~Hz})$ si el promedio es menor a 25 (dB), la audiometría se considera normal, si es mayor se considera con pérdida auditiva o hipoacusia como lo muestra la figura 2. 
Figura 2. Toma de Audiometría Tamizaje

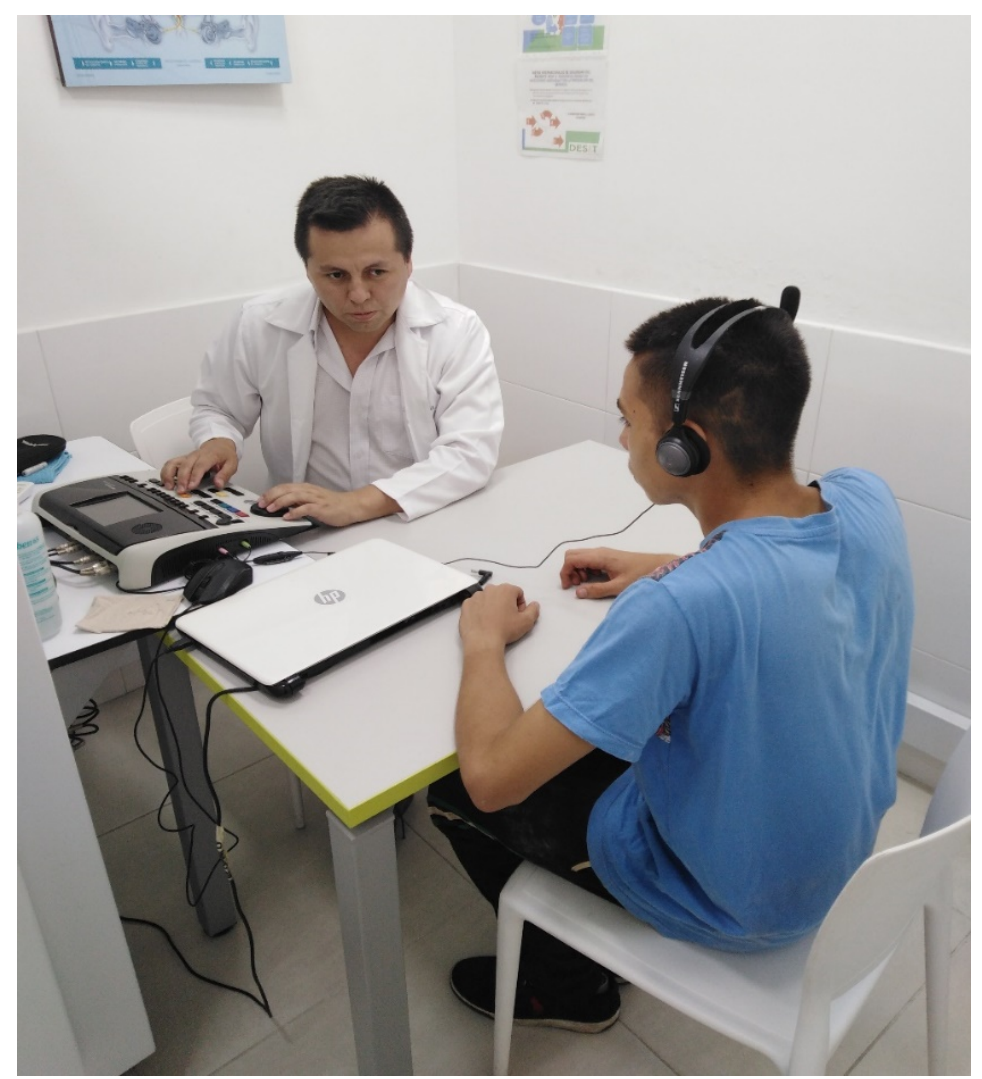

Fuente: Semillero SIVIEPI

La tabulación y análisis de los resultados se utilizó la herramienta del programa estadístico SPSS versión 24 , determinando las medias aritméticas, los porcentajes, el dato mínimo-máximo y el valor - $p$ para establecer la correlación entre el nivel de exposición al riesgo térmico y ruido con la fatiga auditiva reflejado en el comportamiento de la curva audiométrica de cada trabajador. 
Tabla 1. Cronología de los procedimientos realizados con la normatividad

\begin{tabular}{|c|c|c|c|c|}
\hline Hora & Tipo de Medición & Instrumento & $\begin{array}{l}\text { \# de } \\
\text { Trabajadores } \\
\text { tamizados }\end{array}$ & Norma Técnica de medición \\
\hline 7.15 Am & $\begin{array}{l}\text { Sonometría, índice } \\
\text { WBGT, } \\
\text { audiometría Tamiz }\end{array}$ & $\begin{array}{l}\text { Sonómetro } \\
\text { Equipo de Estrés } \\
\text { térmico } \\
\text { Audiómetro }\end{array}$ & 24 & $\begin{array}{l}\text { ISO } 1999 \text { (ruido) } \\
\text { NTC } 322 \text { (estrés térmico) } \\
\text { SAL-AMA (Asociación Médica } \\
\text { americana)- (tamiz auditivo) }\end{array}$ \\
\hline $1.15 \mathrm{Pm}$ & $\begin{array}{l}\text { Sonometría, índice } \\
\text { WBGT, } \\
\text { audiometría Tamiz }\end{array}$ & $\begin{array}{l}\text { Sonómetro } \\
\text { Equipo de Estrés } \\
\text { térmico } \\
\text { Audiómetro }\end{array}$ & 24 & $\begin{array}{l}\text { ISO } 1999 \text { (ruido) } \\
\text { NTC } 322 \text { (estrés térmico) } \\
\text { SAL-AMA (Asociación Médica } \\
\text { americana)- (tamiz auditivo) }\end{array}$ \\
\hline 5.15 Pm & $\begin{array}{l}\text { Sonometría, índice } \\
\text { WBGT, } \\
\text { audiometría Tamiz }\end{array}$ & $\begin{array}{l}\text { Sonómetro } \\
\text { Equipo de Estrés } \\
\text { térmico } \\
\text { Audiómetro }\end{array}$ & 24 & $\begin{array}{l}\text { ISO } 1999 \text { (ruido) } \\
\text { NTC } 322 \text { (estrés térmico) } \\
\text { SAL-AMA (Asociación Médica } \\
\text { americana)- (tamiz auditivo) }\end{array}$ \\
\hline
\end{tabular}

Fuente: Semillero SIVIEPI

\section{RESULTADOS}

En la Tabla 2 se describen las características sociodemográficas de la población objeto de estudio, en la distribución por la edad en la población expuesta con una media de 40 años, en la no expuesta de 36,5 años con un valor -p no significativo.

Tabla 2. Características generales de la población estudiada

\begin{tabular}{lccc}
\hline \multicolumn{1}{c}{ Características } & $\begin{array}{c}\text { Grupo } \\
\text { Expuestos }\end{array}$ & $\begin{array}{c}\text { Grupo } \\
\text { No Expuestos }\end{array}$ & Valor_p \\
EDAD (años) $\mathbf{n}$ & 12 & 12 & 0,164 \\
Media \pm D. estándar & $40 \pm 11,3$ & $36,5 \pm 10,5$ & \\
Rango & $23-64$ & $19-57$ & 0,633 \\
GÉNERO $\mathbf{n}(\%)$ & $12(50)$ & $12(50)$ & \\
Hombre & $9(75)$ & $9(75)$ & 0,000 \\
Mujer & $3(25)$ & $3(25)$ & \\
CARGO $\mathbf{n}(\%)$ & 12 & 12 & \\
Operativo & $12(100,0)$ & $12(100,0)$ & \\
Administrativo & & & \\
\end{tabular}

Fuente: Semillero SIVIEPI

La Tabla 3 muestra las características promedio de las tres mediciones de higiene de ruido temperatura que se realizaron en el trascurso de 10 horas, obteniendo en la sonometría una media $87,5 \mathrm{~dB}$ para la población expuesta, en comparación con la no expuesta de una media 
de 59,7 dB, en el Índice de estrés térmico WBGT con radiación solar utilizando la ecuación establece la norma NTP $322($ WBGT $=0.7 \mathrm{THN}+0.2 \mathrm{TG}+0.1 \mathrm{TA}(\mathrm{II}))$, se encontró para los expuestos una media de $31,4^{\circ} \mathrm{C}$, en los no expuestos una $28,2^{\circ} \mathrm{C}$.

Tabla 3. Características laborales y extra-laborales

\begin{tabular}{|c|c|c|}
\hline Características & Grupo- Expuestos & $\begin{array}{l}\text { Grupo- } \\
\text { Expuestos }\end{array}$ \\
\hline RESULTADOS DE SONOMETRIA (dB) n (\%) & 12 & 12 \\
\hline Media \pm D. estándar & $87,5 \pm 7,93$ & $59,7 \pm 5,76$ \\
\hline Rango & $78,6-98,8$ & $54,3-69,6$ \\
\hline RESULTADOS DE WBGT $\left({ }^{\circ} \mathrm{C}\right) \mathrm{n}(\%)$ & 12 & 12 \\
\hline Media \pm D. estándar & $31,4 \pm 1,29$ & $28,2 \pm 1$ \\
\hline Rango & $29,61-32,96$ & $26,78-29,56$ \\
\hline TIEMPO DE LA JORNADA LABORAL (HORAS) n \% & 12 & 12 \\
\hline Media \pm D. estándar & $8,9 \pm 0,79$ & $8,5 \pm 0,67$ \\
\hline Rango & $8-10$ & $8-10$ \\
\hline ANTIGÜEDAD EN LA EMPRESA (AÑOS) n \% & 12 & 12 \\
\hline Media \pm D. estándar & $2,5 \pm 1,2$ & $4 \pm 2,35$ \\
\hline Rango & $1-5$ & $1-7$ \\
\hline
\end{tabular}

Fuente: Semillero SIVIEPI

\section{Tamizajes audiométricos según los criterios de la OSHA}

En el análisis del comportamiento auditivo se partió de una audiometría base que se realizó a los 24 trabajadores de la mina, aunque se evaluaron toda la gama de frecuencia, para la importancia del estudio, solo se va tener presente las frecuencias que según los criterios de la Occupational Safety and Health Administration (OSHA), tienen mayor predisposición al cambio del umbral auditivo por la exposición constante al ruido que son las frecuencias de 2000, 3000 y $4000 \mathrm{~Hz}$.

En la Tabla 4 se muestra los resultados obtenidos en el primer tamizaje de dichas frecuencias, para ello, se utilizó el segundo criterio de la OSHA, en la que afirma que el umbral auditivo normal para cualquiera de las 8 frecuencias que se evalúan debe ser igual o menor de $\geq 25 \mathrm{~dB}$ para considerarse normal. En la primera medición no se observa datos significativamente relevantes entre la población expuesta y no expuestas a los factores de riesgos ruido y estrés térmico. En la frecuencia de $4000 \mathrm{~Hz}$ en la primera medición se percibe que el $41,7 \%$ de los trabajadores expuestos presentan una pérdida de la audición por encima de los $25 \mathrm{~dB}$ para el 
oído derecho, mientras en el oído izquierdo un 33, 3\% de los trabajadores, sin embargo, en los trabajadores no expuestos el $16,7 \%$ presentan la perdida por encima de $25 \mathrm{~dB}$ en el oído derecho, en el oído izquierdo $8,3 \%$ de los trabajadores.

Se aprecia los resultados obtenidos del segundo tamizaje que se realizó 6 horas después, utilizando el mismo criterio OSHA de la primera medición; no se encontró un valor _p estadísticamente significativo entre los dos grupos, solo un aumento del porcentaje de caída en la frecuencia de $4000 \mathrm{~Hz}$ en el oído derecho del 50\% del grupo expuesto con aumento del nivel de ruido en la sonometría superior por 36,6 dBA, en el índice de estrés térmico superior por $6,2^{\circ} \mathrm{C}$ en comparación con el grupo no expuesto. En la tercera medición realizada 10 horas después de la primera, se observa en la Tabla 6, qué en el grupo de expuestos el promedio de caída $\leq 25 \mathrm{~dB}$, se mantiene igual que la medición anterior en el oído derecho, un leve aumento del promedio en el oído izquierdo en la frecuencia de $4000 \mathrm{~Hz}$ que paso $8,3 \%$ de trabajadores con caída por encima de $25 \mathrm{~dB}$ a un $16,7 \%$ de trabajadores, en el grupo control de no expuestos los datos son similares a la medición anterior.

En el análisis de los cambios del umbral de la audición de la población expuesta a ruido por encima de los $88,5 \mathrm{dBA}$ y con un promedio de índice de estrés térmico de $31,6^{\circ} \mathrm{C}$ durante 10 horas de seguimiento, se calculó una media aritmética de los resultados obtenidos en las tres mediciones de las audiometrías, para su apreciación se realizó la figura 2. Con las tres curvas, el comportamiento de los umbrales durante primera hora de exposición y la sexta hora de la jornada laboral se mantiene dentro del rango de la normalidad por debajo de los $25 \mathrm{~dB}$, con pequeñas fluctuaciones de 3 a $5 \mathrm{~dB}$ en toda la gama de frecuencias; en la tercera medición que se realizó después de 10 horas de trabajo se aprecian cambios significativos en las frecuencias agudas de 6000 y $8000 \mathrm{hz}$ con un aumento de caída del umbral de 8 a $10 \mathrm{~dB}$ entre la primera medición y la última medición.

Figura 2. Comportamiento umbral auditivo de la población expuesta

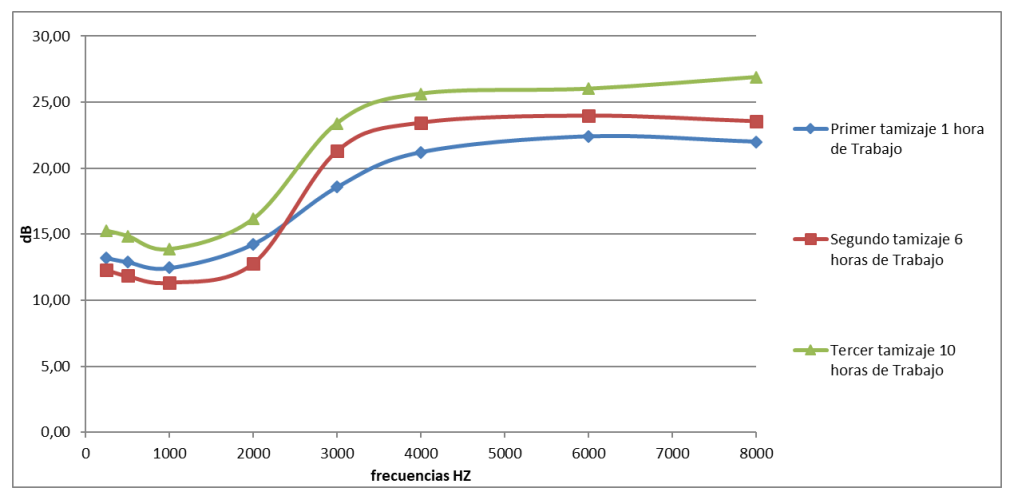

Fuente: Semillero SIVIEPI

En la población no expuesta el comportamiento del umbral auditivo durante las 10 horas de seguimiento no presenta ninguna fluctuación significativa durante las tres mediciones realizadas de la audición como lo muestra la Figura 3.

Figura 3. Comportamiento umbral auditivo de la población no expuesta 


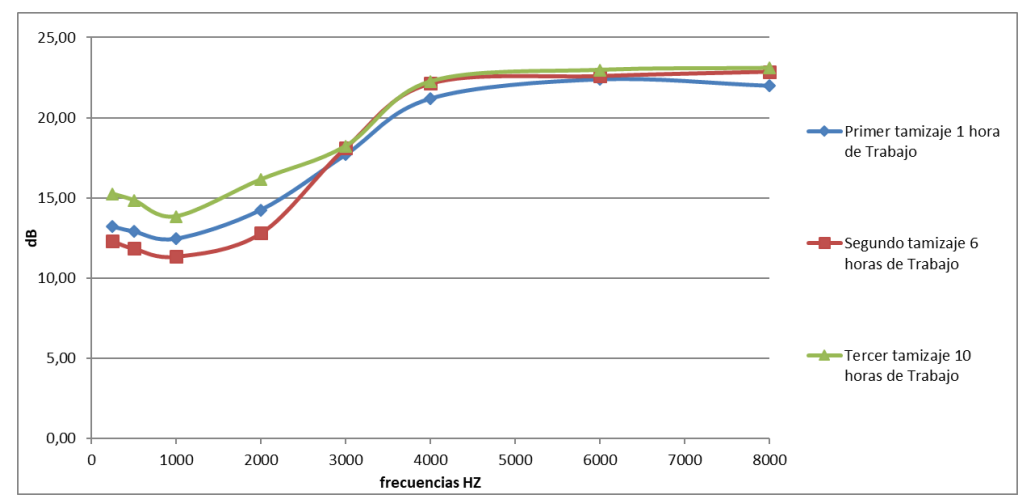

Fuente: Semillero SIVIEPI

\section{CONCLUSIONES}

El estudio del comportamiento del umbral auditivo en trabajadores expuestos y no expuestos ruido y altas temperaturas durante 10 horas de seguimiento con tres mediciones, permite determinar las siguientes conclusiones:

El grupo expuesto y no expuesto en la primera medición realizada a las 7 am de la jornada laboral, no se determinó diferencias estadísticamente significativas, solo una relación de caída del umbral auditivo por encima de los 25 decibeles 1 a 3 en el oído derecho y 1 a 2 en el oído izquierdo con mayor predominio de perdida en los trabajadores expuestos. La anterior diferencia se puede generar porque el grupo de expuestos tienen un promedio de antigüedad en el cargo de 2 a 5 años, tiempo suficiente para desarrollar una caída auditiva en las frecuencias agudas en especial la de $4000 \mathrm{hz}$ como lo establece los diferentes estudios académicos, otro factor determinante de dicho comportamiento es la edad del grupo expuesto que es mayor que la no expuestos con un promedio de 40 años, según la propedéutica de la etiología de la presbiacusia edad propicia desarrollar pérdida auditiva en la frecuencia $4000 \mathrm{~Hz}$. En las diferentes investigaciones realizadas por teóricos Azoy y Maduro que realizaron una serie de experimentos con soldados de la segunda Guerra Mundial; se estableció cuatro fases de la Hipoacusia Inducida por Ruido (HIR), en la primera fase I (de instalación de un déficit permanente). "Antes de la instauración de una HIR irreversible se produce un incremento del umbral de aproximadamente $30-40 \mathrm{~dB}$ en la frecuencia $4 \mathrm{kHz}$ ", un tiempo de exposición aproximado de 5 años.

Trittipoe (1958) citado por Salazar (2012) describió el fenómeno del desplazamiento del umbral auditivo de forma temporal y permanente afirma:

Cuando una persona se expone a niveles de ruido elevados durante un intervalo de tiempo prolongado, puede llegar a producirse un desplazamiento temporal del umbral de audición Este desplazamiento consiste en una elevación del "nivel umbral' causado por la presencia de ruido, produciéndose posteriormente una recuperación total de la capacidad auditiva al cabo de un tiempo.

En la segunda y tercera medición en el grupo expuestos el comportamiento auditivo se observa correlación significativa entre el aumento del nivel de ruido (NPSeq $\geq 90 \mathrm{~dB}$ ), el aumento del índice de estrés térmico (WBGT $\geq 31,2^{\circ} \mathrm{C}$ ) con los cambios del umbral auditivo en las frecuencias 
de 3 a $8 \mathrm{~Hz}$, En la sexta hora de la jornada laboral el umbral auditivo empieza aumentar la caída de 2 a $3 \mathrm{~dB}$ en comparación con la primera medición, y en la tercera medición a decima hora de la jornada laboral la caída del umbral auditivo pasa de 4 a $8 \mathrm{~dB}$ en comparación con la audiometría Base. Explicación que se puede desencadenar por la fatiga auditiva, vasoconstricción periférica como lo describen los diferentes autores consultados en el estudio. En el grupo de control (no expuestos) no se encontró ninguna diferencia significativa en las tres mediciones.

El estudio resulta novedoso porque incluye una serie de mediciones higiene del ruido y el estrés térmico con las audiometrías tamiz en una jornada laboral comparando dos grupos poblacional, uno expuesto y otro no expuestos, obteniendo diferencias en el comportamiento del umbral auditivo entre los dos grupos en las frecuencias agudas, el estudio debe continuar teniendo presentes otros factores de riesgos como las vibraciones, y las radiaciones no ionizantes.

Puede existir una correlación entre los factores de riesgo ruido y la temperatura con los cambios del umbral auditivo en las frecuencias agudas, ya que la intensidad de los estímulos sonoros en forma de ruido continuo y el aumento del estrés térmico producen vasoconstricción periférica que disminuye el nivel concentración en los trabajadores, genera fatiga auditiva, aumento de los incidentes y accidentes laborales como lo demuestras diferentes investigaciones en sectores industriales con altos niveles de ruido y de temperatura (Urban, 1985; citado por (Hernández \& González, 2008, p. 2).

\section{REFERENCIAS}

1. García, I. P. (2007). Ambientes laborales de ruido en el sector minero de la Comunidad de Madrid: clasificación, predicción y soluciones. Madrid: Tesis (Doctoral), E.T.S.I. Industriales (UPM).

2. Hernández, A., \& González, B. M. (2008). Alteraciones auditivas en trabajadores expuestos al ruido industrial. Medicina y Seguridad del Trabajo, 1-11.

3. JA., M. (1969). Ruido y sordera: Sordera profesional por ruido. Salamanca: Ed. Grafiseca.

4. López, J. M. (1992). Validez de los tests predictivos de la fatiga auditiva en la prevención del trauma acústico. Madrid: Facultad de Medicina de la Universidad Complutense.

5. Organización Mundial de la Salud. (2019). Sordera y pérdida de la audición.

Ginebra: OMS. Rosen, G. (1974). A backward glance at noise pollution.

American Journal of Public Health, 574-

a. 577.

6. Salazar, A. M. (2012). Pérdida auditiva por contaminación acústica laboral en Santiago de Chile.

a. Barcelona: Universidad de Barcelona. 
7. Silva, K. A. (2013). Características audiológicas en trabajadores de una fábrica textil de la ciudad Santiago. Santiago, Chile: Universidad Andrés Bello.

8. Soto, J. L. (2010). Evaluación y prevención de riesgos en calderas de generación eléctrica. Quito: Tesis de Maestría; Universidad San Francisco de Quito universidad de Huelva España. 